\title{
BMJ Open Memory for gist and detail information in patients with Parkinson's disease
}

\author{
Rwei-Ling Yu, ${ }^{1}$ Chun-Hsiang Tan, ${ }^{2}$ Yih-Ru Wu, ${ }^{3}$ Ruey-Meei Wu, ${ }^{4}$ Ming-Jang Chiu, ${ }^{4}$ \\ Mau-Sun Hua ${ }^{4,5,6,7,8}$
}

To cite: Yu R-L, Tan C-H, Wu Y-R, et al. Memory for gist and detail information in patients with Parkinson's disease. BMJ Open 2015;5: e009795. doi:10.1136/ bmjopen-2015-009795

\section{- Prepublication history for this paper is available online. To view these files please visit the journal online (http://dx.doi.org/10.1136/ bmjopen-2015-009795).}

Received 21 August 2015 Revised 26 October 2015 Accepted 27 October 2015

CrossMark

For numbered affiliations see end of article.

\section{Correspondence to} Dr Mau-Sun Hua; huams@ntu.edu.tw

\section{ABSTRACT}

Objective: Memory formation is proposed to be a dual process that involves the simultaneous memorisation of both detailed information (itemspecific memory) and gist information (gist memory). Memory deficits have been reported in patients with Parkinson's disease (PD); however, few studies have explicitly addressed the nature of these deficits. To obtain a detailed understanding of memory dysfunction in patients with $\mathrm{PD}$, it is of crucial importance to establish whether item-specific memory and gist memory performance are impaired. The aim of this study is to explore whether gist memory and itemspecific memory performance are still intact in patients with $\mathrm{PD}$, as well as to determine which psychological mechanisms are responsible for memory formation.

Setting: Two hospitals in northern Taiwan.

Participants: Thirty-nine patients with PD and 28 normal controls were recruited. Each participant received a gist-based recognition test following the Deese-Roediger-McDermott paradigm, as well as neuropsychological tests and measures of clinical characteristics.

Results: Gist memory was impaired in patients with advanced-stage disease (Hoehn and Yahr (H\&Y) stage: III) $\left(F_{2,64}=3.58, p=0.033\right)$, whereas item-specific memory was preserved throughout all disease stages. Correlation analysis showed that item-specific memory was related to executive functions in normal controls and early-stage patients with PD (H\&Y stage: I-II); however, item-specific memory was related to episodic memory, rather than to executive functions, in advanced-stage patients with PD. Moreover, gist memory was related to episodic memory, but only in early-stage patients with PD.

Conclusions: We discovered that impaired gist memory is found in advanced-stage, but not in earlystage, patients with PD. Our findings suggest that the techniques used to take advantage of the relatively preserved gist memory in early-stage patients with PD, as well as the preserved item-specific memory in patients with PD of all stages, could be useful for memory rehabilitation programmes.

\section{INTRODUCTION}

Parkinson's disease (PD) is a neurodegenerative disease that is pathologically

\section{Strengths and limitations of this study}

- This study offers a cross-disciplinary approach to explore non-motor function in patients with Parkinson's disease (PD).

- We expand on the current psychological knowledge (item-specific and gist memory) in clinical research.

- We find that gist memory is impaired in patients with advanced-stage PD, while item-specific memory is preserved.

- We propose that gist memory might be related to episodic memory abilities among early-stage patients with PD, whereas item-specific memory might also be related to episodic memory in advanced-stage patients with PD.

- Our study featured a small sample size of advanced-stage patients, which may affect the generalisability of our findings.

characterised by the loss of dopaminergic neurons in the substantia nigra, and it manifests clinically as resting tremor, rigidity, bradykinesia and postural instability. In light of the close connections between the basal ganglia and prefrontal cortex, ${ }^{1}$ growing evidence has shown that non-motor symptoms may also be present in patients with PD, including neurocognitive dysfunction ${ }^{2}$ and impairments in social cognitive function. ${ }^{3} 4$ Neurocognitive impairment is one of the most common and devastating non-motor symptoms of PD. ${ }^{2}$ The evidence shows that the mean prevalence of mild cognitive impairment is $27 \%$ (range: $19-38 \%$ ), and that the prevalence of dementia in PD is more than $30 \% ;^{5}$ around $60 \%-78 \%$ of patients with PD ultimately develop dementia by the end of longer follow-up periods. ${ }^{6} 7$ Memory dysfunction is frequently found in association with $\mathrm{PD},{ }^{2}$ and the status of recognition memory in PD is controversial. Early studies have suggested that recognition is intact in $\mathrm{PD}^{8}$ nevertheless, a meta-analysis has shown that recognition can be significantly impaired in PD. ${ }^{9}$ 
The process of memory formation is complicated, and single-process models are insufficient at explaining the process fully; therefore, the dual-process model of recognition memory was developed. Fuzzy trace theory (FTT) is a widely accepted theory that is used to explain the process of memory construction. ${ }^{10}$ According to FTT, memory formation involves the simultaneous operation of dual processes that consist of the memorisation of detailed information (item-specific memory) and gist information (gist memory). Item-specific memory refers to one's memory for the specific or superficial features of a stimulus, whereas gist memory involves well-integrated tracking of the features that are shared among stimuli. ${ }^{11}$ On the basis of this framework, false memories may arise from an involvement of gist information, rather than from the verbatim trace used to recall certain information or from a disintegration of the two traces. Roediger and McDermott $(1995)^{12}$ modified Deese's ${ }^{13}$ experiment and developed the Deese-Roediger-McDermott (DRM) paradigm, which enabled the false recognition effect to be replicated and employed to investigate item-specific, and gist memory performance, in normal and clinical populations (eg, in cases of epilepsy; ${ }^{14}$ mild cognitive impairment (MCI) $;{ }^{15}$ Alzheimer's disease (AD) $;{ }^{16}$ and frontal lobe damage). ${ }^{16-18}$

Memory for gist and item-specific information may require qualitatively distinct underlying psychological processes. ${ }^{12}$ The encoding of gist information may partly rely on automatic activation processes; these are the processes through which activation of the familiarity of an item spreads to related concepts. In contrast, item-specific memory may depend on controlled/effortful activation processes. The possible underlying neural substrates for gist memory have been associated with the mesial temporal structures, ${ }^{14}{ }^{17-21}$ whereas the frontal lobes and adjacent structures, as well as the hippocampal gyrus might be engaged in the processing of item-specific memory. ${ }^{16} 17{ }^{21-24}$ Some investigators have proposed that episodic memory is required for participants to encode and memorise the general meaning of a list of related words $;{ }^{1525}$ however, other empirical evidence suggests that executive functions may contribute to the reduction of the false recognition effect. ${ }^{16}$ Executive functions, which include monitoring and inhibition, are essential roles that are played by the frontal lobe. Normally, the frontal lobe exerts an inhibitory influence on the posterior cortical regions, particularly on the temporal lobes, through reciprocal connectivity of the frontotemporal circuits. Accordingly, an increase in gist-based memory may require activation of the mesial temporal structures resulting from an attenuation of frontal inhibitory input due to frontal dysfunction.

Although there is a growing consensus that dualprocess models provide a good description of memory function, only a few studies have been conducted with PD populations, and these have produced conflicting evidence. ${ }^{26}{ }^{27}$ Specifically, researchers have found that patients with PD showed diminished detail and context recognition memory, ${ }^{27}$ and it was suggested that this reduction in recognition is primarily due to an impairment in familiarity rather than to a decline in one's memory for detail. ${ }^{26}$ A separate study observed intact recognition in patients with $\mathrm{PD}$; however, mild to moderately affected patients with PD were selectively impaired in terms of familiarity, but their memory for detail was intact. ${ }^{28}$ Gaining a deeper understanding of the neuropsychological mechanisms underlying the gist-based memory framework will aid in our overall understanding of how memories are formed, and it may lead to future strategies for memory remediation in patients with memory deficits. However, to our knowledge, no clinical studies have investigated the gist-based memory framework using the traditional psychological approach-the DRM paradigm-in patients with PD. Furthermore, the underlying cognitive processes that mediate gist and item-specific memory remain unclear. Thus, the specific goals of this study are to examine the following issues: (1) are patients with PD more vulnerable to gist-based memory dysfunction when compared to normal participants? (2) Are there other associated cognitive processes that contribute to gist-based memory?

\section{METHODS}

\section{Participants}

Thirty-nine patients who had been diagnosed with idiopathic PD according to the UK PD Society Brain Bank clinical diagnostic criteria, ${ }^{29}$ as well as 28 community-based, normal control participants (NCs), were enrolled in this study. The patients with PD were recruited via referrals from neurologists at the Movement Disorders Centre. Each patient was assessed while 'ON' medication and the patients' motor disability ranged from mild to moderate severity, according to the Hoehn and Yahr (H\&Y) staging criteria $^{30}$ (29 early-stage PD (H\&Y stage: I-II) and 10 advanced-stage PD (H\&Y stage: III)). The exclusion criteria included the following: illiteracy, a history of a brain operation, atypical parkinsonism (including dementia with Lewy bodies, progressive supranuclear palsy and multiple system atrophy), mood disorders (including depressive and anxiety disorders), psychosis, severe systemic disease and global cognitive deterioration (Mini-Mental State Examination (MMSE) <24). ${ }^{31}$ All participants provided written informed consent prior to enrolment, in accordance with the ethical standards set forth in the Declaration of Helsinki (1964). This study was approved by the ethics committees of the participating hospitals.

\section{Gist-based recognition task}

The design of this task was modified from the DRM paradigm $^{12}$ and the details have been published elsewhere by our group. ${ }^{14}$

\section{MATERIALS AND DESIGN}

Twelve semantically related word lists were selected from the Chinese word association norms. Each list contained 
12 words; 12 words were presented during the learning phase in order of decreasing associative strength to the non-presented (but related) lure word. The 'critical lure' was not shown during the learning phase. For counterbalancing purposes, a total of 12 lists were divided into three sets of 4 lists. Every participant had an equal chance of using each word list group. Every participant was presented with 8 lists. The 36 words used in the recognition phase were obtained from the 12 lists, and the selected words consisted of 16 presented items in the studied list (targets), 8 items in the unstudied list (target distracters), 8 lure items from the non-presented critical lure in each studied list and 4 items from the critical lure of each unstudied list (lure distracters). These 36 words were divided into two groups, and the words in the same group were displayed in a randomised sequence.

During the learning phase, all participants were instructed to read and memorise the words displayed on the screen and they were reminded that their memories for these items would be tested later. Words from a given list were displayed sequentially with no break provided between different lists. We then performed the orientation task (ie, orientation to time and place) before the recognition phase, which took approximately $3 \mathrm{~min}$ to complete; this was used as a filler task (retention phase). During the recognition phase, participants were instructed to recognise the words shown on the screen, and they were allowed to make decisions at their own pace. The participants then judged whether each item was 'old' (ie, it had been shown previously, during the learning phase) or 'new', by pressing the key labelled 'old' or 'new', respectively. Each word was displayed at the centre of a 15-inch LCD screen using the DMDX software programme on a laptop, featuring a reading distance of approximately $60 \mathrm{~cm}$.

We separately calculated the probabilities with which participants gave 'old' responses for targets, target distracters, critical lures and lure distracters. In order to avoid the participants' response bias, we computed the corrected target recognition rate by subtracting the number of 'old' responses for target distracters from the number of 'old' responses for targets; we also calculated the corrected lure recognition rate by subtracting the number of 'old' responses for lure distracters from the number of 'old' responses for critical lures for each subject. We defined the corrected lure recognition rate as the gist memory of the DRM paradigm; item-specific memory was defined by subtracting the number of 'old' responses for critical lures from the number of 'old' responses for targets.

\section{Executive function}

The Modified Card Sorting Test (MCST), ${ }^{32}$ the semantic ('fruit', 'fish' and 'vegetable' categories) word association component of the Verbal Fluency (VF) Test $^{33}$ and the Trail Making Test (TMT), ${ }^{34}$ were applied. The numbers of achieved categories and perseverative errors were used as MCST indices. For the VF Test, participants were asked to report as many items as possible within each category, in $1 \mathrm{~min}$. The score was a summation of the participants' responses in those categories. The TMT scores are reported as the number of seconds required to complete the task; therefore, higher scores reveal greater impairment.

\section{Memory function}

The Logical Memory subtest of the Taiwan version of the Wechsler Memory Scale-Third Edition (WMS-III) was used to measure various memory functions, including immediate and delayed recall and recognition. ${ }^{35}$ Both immediate and delayed recall consisted of scores for free recall and thematic recall units.

\section{Statistical analysis}

The data were examined for normality and homogeneity of variance. The $\chi^{2}$ test, Mann-Whitney $U$ test and Kruskal-Wallis test, were used to examine group differences in non-parametric variables. Furthermore, $t$ tests and one-way analysis of variance (ANOVA) were performed. For all significant effects (determined by an $\alpha$-level of 0.05), post hoc Fisher's Least Significant Difference (LSD) tests were applied. To examine the potential relationships between variables, we calculated Pearson's correlation coefficients (for parametric data) and Spearman's rank correlations (for non-parametric data). The analyses were carried out with SPSS V.17.0 for Windows (SPSS, Inc, Chicago, Illinois, USA).

\section{RESULTS}

\section{Clinical and neuropsychological background}

The demographic and clinical data are summarised in table 1. The three experimental subgroups did not differ in terms of age or gender. The subgroups of patients with PD were also equated according to their age at onset; however, those in the advanced-stage PD group had longer disease duration than those in the early-stage PD group ( $\mathrm{U}=47.5, \mathrm{p}=0.012)$. Patients scored significantly lower on all measures of the Logical Memory subtest (immediate free $(p=0.001)$ and theme recall $(\mathrm{p}=0.001)$ scores, as well as on the delayed free $(\mathrm{p}<0.0001)$ and theme recall $(\mathrm{p}<0.0001)$ scores, and recognition scores $(p=0.005))$. Regarding the executive function tests, a significant difference was found between the study groups on the MCST (achieved categories, $\mathrm{p}<0.0001$; perseverative errors, $\mathrm{p}=0.002)$ ) ; moreover, advanced-stage patients with PDperformed significantly worse than the NCs on the VF Test $(\mathrm{p}=0.008)$.

\section{Gist versus item-specific memory}

As shown in table 2 and figure 1, after correcting for response bias, the ANOVA revealed a significant group difference in gist memory $\left(\mathrm{F}_{2,64}=3.58, \mathrm{p}=0.033\right)$. Post hoc tests indicated that the advanced-stage PD group had the worst gist memory performance. No significant 
Table 1 Demographic, clinical characteristics and neuropsychological function in study groups *

\begin{tabular}{|c|c|c|c|}
\hline & \multirow[b]{2}{*}{ NCs $(n=28)$} & \multicolumn{2}{|l|}{ PD } \\
\hline & & Early stage $(n=29)$ & Advanced stage $(n=10)$ \\
\hline Age (years) & $66.64 \pm 6.18$ & $62.55 \pm 8.77$ & $64.70 \pm 11.20$ \\
\hline Gender (male/female) & $17 / 11$ & $18 / 11$ & $9 / 1$ \\
\hline Age at onset (years) & - & $56.29 \pm 9.36$ & $54.39 \pm 13.17$ \\
\hline Disease duration (years) & - & $5.08 \pm 2.54$ & $8.72 \pm 3.80 \dagger$ \\
\hline MMSE & $27.29 \pm 1.58$ & $26.4 \pm 1.43 \ddagger$ & $25.90 \pm 1.29 \ddagger$ \\
\hline \multicolumn{4}{|l|}{ WMS-LM } \\
\hline immediate-recall (raw) & $33.46 \pm 10.99$ & $23.96 \pm 10.43 \ddagger$ & $17.55 \pm 8.05 \ddagger$ \\
\hline immediate-theme (raw) & $14.43 \pm 4.28$ & $11.17 \pm 4.28 \ddagger$ & $8.25 \pm 4.20 \ddagger$ \\
\hline delayed-recall (raw) & $20.68 \pm 8.37$ & $11.63 \pm 7.40 \ddagger$ & $10.38 \pm 6.39 \ddagger$ \\
\hline delayed-theme (raw) & $9.50 \pm 3.58$ & $5.88 \pm 3.79 \ddagger$ & $4.88 \pm 3.18 \ddagger$ \\
\hline recognition & $24.11 \pm 3.11$ & $22.08 \pm 3.74 \ddagger$ & $19.88 \pm 2.36 \ddagger$ \\
\hline \multicolumn{4}{|l|}{ Executive function } \\
\hline Verbal Fluency & $36.93 \pm 6.76$ & $35.36 \pm 8.74$ & $28.10 \pm 5.02 \dagger \ddagger$ \\
\hline MCST-C & $4.86 \pm 1.88$ & $3.59 \pm 1.68 \ddagger$ & $2.10 \pm 1.37 \dagger \neq$ \\
\hline MCST-P & $4.39 \pm 4.43$ & $8.41 \pm 7.35 \ddagger$ & $12.70 \pm 9.88 \ddagger$ \\
\hline TMTA & $69.19 \pm 37.33$ & $70.70 \pm 40.37$ & $113.40 \pm 53.92 \dagger \ddagger$ \\
\hline TMTB & $136.83 \pm 53.38$ & $181.04 \pm 127.7$ & $368.33 \pm 230.02 \dagger \ddagger$ \\
\hline
\end{tabular}

\begin{tabular}{|c|c|c|c|}
\hline & \multirow[b]{2}{*}{$\begin{array}{l}\text { NCs } \\
(n=28)\end{array}$} & \multicolumn{2}{|l|}{ PD } \\
\hline & & $\begin{array}{l}\text { Early } \\
(n=29)\end{array}$ & $\begin{array}{l}\text { Advanced } \\
(n=10)\end{array}$ \\
\hline \multicolumn{4}{|l|}{ List targets } \\
\hline Studied list† & $0.81 \pm 0.15$ & $0.64 \pm 0.23 \ddagger$ & $0.69 \pm 0.20$ \\
\hline Unstudied list & $0.15 \pm 0.17$ & $0.16 \pm 0.21$ & $0.20 \pm 0.12$ \\
\hline $\begin{array}{l}\text { CTR (studied- } \\
\text { unstudied)§ }\end{array}$ & $0.66 \pm 0.19$ & $0.48 \pm 0.23 \ddagger$ & $0.49 \pm 0.15 \ddagger$ \\
\hline \multicolumn{4}{|l|}{ Critical lure } \\
\hline Studied list & $0.65 \pm 0.20$ & $0.55 \pm 0.25$ & $0.54 \pm 0.24$ \\
\hline Unstudied list & $0.20 \pm 0.21$ & $0.10 \pm 0.17$ & $0.35 \pm 0.38$ \\
\hline $\begin{array}{l}\text { Gist memory } \\
\text { (studied lure- } \\
\text { unstudied } \\
\text { lure)§ }\end{array}$ & $0.46 \pm 0.25$ & $0.45 \pm 0.30$ & $0.19 \pm 0.35 \neq \rrbracket$ \\
\hline $\begin{array}{l}\text { Item-specific } \\
\text { memory (studied } \\
\text { target-studied } \\
\text { lure) }\end{array}$ & $0.16 \pm 0.20$ & $0.09 \pm 0.22$ & $0.16 \pm 0.23$ \\
\hline $\begin{array}{l}\text { Unstudied target } \\
\text { +unstudied lure }\end{array}$ & $0.35 \pm 0.36$ & $0.26 \pm 0.35$ & $0.55 \pm 0.38$ \\
\hline \multicolumn{4}{|c|}{ 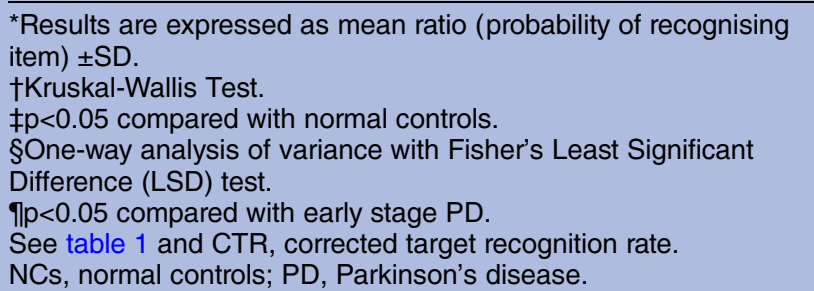 } \\
\hline
\end{tabular}

difference in item-specific memory was found between the three groups. Furthermore, there was a significant group effect $\left(\mathrm{F}_{2,64}=6.00, \mathrm{p}=0.004\right)$ for the corrected target recognition rate (studied-unstudied). At the individual level, there was a significant intra-subject difference in the performance of item-specific memory relative to that of gist memory in the DRM paradigm among early-stage patients with PD (paired t test, t(28) $=4.13, \mathrm{p}<0.0001)$ and NCs $(\mathrm{t}(27)=4.23, \mathrm{p}<0.0001)$.

\section{Correlations between the gist-based recognition task and cognitive function}

There was no significant correlation between scores on the gist-based task (gist memory and item-specific memory) and disease duration in the two patient groups (see table 3). The correlations between variables were computed separately for the three population groups. Gist memory was positively associated with the scaled score of the LM-I free recall $(r=0.52, p=0.01)$ in the early-stage PD group, while item-specific memory was positively correlated with the LM-II thematic $(r=0.73$, $\mathrm{p}=0.01$ ). Nevertheless, no significant correlation was found in the advanced-stage PD group.

\section{DISCUSSION}

In this study, the corrected target recognition rate, gist memory performance and item-specific memory performance of older adults, were compatible with previously published results. ${ }^{14-16}$ An important finding is that 


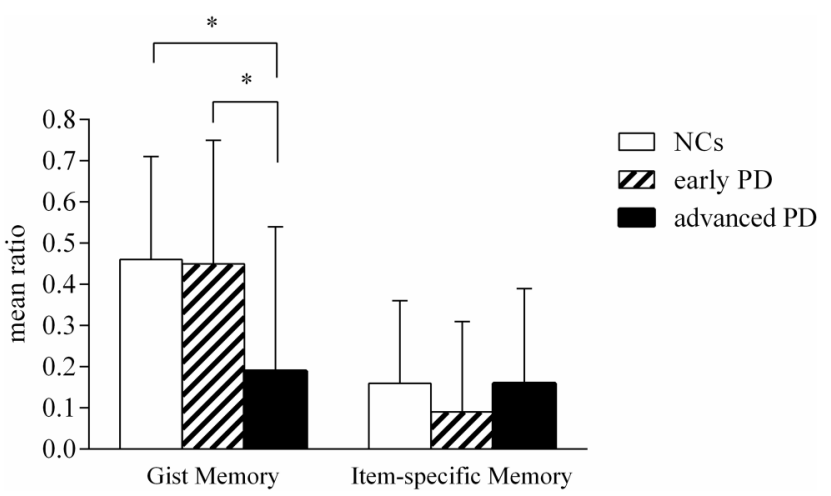

Figure 1 Gist and item-specific memory performances among the study groups $\left({ }^{*} p<0.05\right)$. The advanced-stage PD group exhibited the worst gist memory performance; no significant difference in item-specific memory performance was found between the three groups. NC, normal controls; PD, Parkinson's disease.

advanced-stage patients with PD had severe gist memory impairment, whereas the gist memory performance among early-stage patients with PD was comparable to that of NCs. This result is partially consistent with previous studies reporting that patients with neurodegenerative disease demonstrated a decline in gist memory performance, similar to what has been found among patients with $\mathrm{MCI}^{15}$ and $\mathrm{AD} .{ }^{16}$ In contrast to previous studies on $\mathrm{PD},{ }^{26-28}$ these data suggest that not all patients with PD have impaired gist memory and that early-stage patients with PD are better able to recall gist information than they are detailed information. This indicates that the superiority of gist memory over itemspecific memory found in normal elderly individuals is preserved in early-stage patients with PD. Our study emphasises disease heterogeneity among PD populations, and the results of this study suggest that the ability to develop gist representations from associative information gradually declines as PD progresses. The advancedstage patients with PD identified fewer semantically unrelated words than did the other two groups, suggesting that there was an abnormality in the organisation of the semantic network in advanced-stage patients with PD. According to the dual-process model, our early-stage patients with PD may use both gist and item-specific memory to process memory information, while gist memory was vulnerable in advanced patients with PD. The present findings corroborate and expand on the previous research in terms of overall recognition memory performance in patients with PD. Our patients with PD recognised fewer studied items (as measured by the corrected target recognition rate) relative to healthy controls. These findings are compatible with the previous literature $;^{9}{ }^{27}$ thus, we suggest that patients with PD have impaired recognition memory.

The second aim of the present study was to examine the underlying cognitive processing of gist and itemspecific memory in patients with PD. With respect to the relationship between the corrected target recognition rate, gist memory and item-specific memory, a series of correlation analyses revealed a similar pattern in NCs and early-stage patients with PD: there was a positive correlation between the corrected target recognition rate and gist memory, and a negative correlation between gist memory and item-specific memory. Among the earlystage patients with PD and NCs, enhanced gist memory performance was correlated with a higher corrected target recognition rate. However, this association did not apply to advanced-stage patients with PD. These patients showed no association between the corrected target recognition rate, gist memory and item-specific memory. The association varied within each group, raising the possibility that PD participants with different degrees of disease severity may apply different cognitive resources within the framework of the dual-process model.

Regarding the individual contributions of memory and executive function on gist and item-specific memory performance, in ageing and patients with $\mathrm{PD}$ with varying degrees of disease severity in our ageing population, and early-stage patients with PD, item-specific memory was significantly related to executive function, particularly the flexibility of cognition. This is compatible with previous studies and suggests that item-specific memory is closely related to frontal lobe function, ${ }^{17} 1821^{24}$ and gist memory is mainly related to mesial temporal lobe function. ${ }^{14}$ 17-21 $^{17}$ Hower, our advanced-stage patients with PD appeared to rely on their mesial temporal function, rather than on frontal lobe function, which is responsible for item-specific memory. No associations were found between gist memory performance and other neuropsychological functions in the ageing population. The nature of the task used in this study might account for this finding because the DRM paradigm-a recognition task employed in this study - was less effortful than the recall task. These intergroup differences indicate that gist and item-specific memory depend on distinct cognitive processes, which vary according to the nature of the specific population being studied. We suggest that early-stage patients with PD require greater psychological abilities than the normal ageing population when generating gist memory. Our findings are compatible with those of previous studies, suggesting that the underlying neural substrates responsible for gist memory may be related to temporal structures. ${ }^{14} 1^{17-21}$ It seems that the relatively preserved temporal lobe-related memory function in our patients with PD enabled this group to generate or maintain item-specific memory; this compensatory effect ultimately prevented this memory function from completely breaking down in these patients. However, as the disease progressed, the advanced patients with PD suffered from the deterioration of cortical function to an even greater degree, and their processing capacitywhich is normally provided by the mesial temporal cortices for gist memory-was significantly reduced. Subsequently, this aspect of memory function exhibited a deficit as a result of the mesial temporal cortices 
Table 3 Correlations between gist-based memory and cognitive function in three groups

\begin{tabular}{|c|c|c|c|c|c|c|}
\hline & \multicolumn{2}{|c|}{ NCs $(n=28)$} & \multicolumn{2}{|c|}{ Early PD (n=29) } & \multicolumn{2}{|c|}{ Advanced PD $(n=10)$} \\
\hline & Gist & Item & Gist & Item & Gist & Item \\
\hline Disease duration & - & - & -0.27 & 0.16 & -0.44 & 0.42 \\
\hline \multicolumn{7}{|l|}{ DRM paradigm } \\
\hline CTR & $0.62^{*}$ & 0.30 & $0.54^{*}$ & 0.10 & -0.37 & 0.35 \\
\hline Gist & - & $-0.41 \dagger$ & - & $-0.63^{\star}$ & - & -0.28 \\
\hline Item & $-0.41 \dagger$ & - & $-0.63^{\star}$ & - & -0.28 & - \\
\hline \multicolumn{7}{|l|}{ Memory function } \\
\hline LMI-free recall units & 0.22 & -0.24 & $0.48 \dagger$ & -0.03 & -0.19 & 0.64 \\
\hline LMI—thematic units & 0.25 & -0.30 & 0.32 & 0.03 & -0.14 & $0.73 \dagger$ \\
\hline LMII-free recall units & 0.09 & -0.18 & 0.32 & -0.07 & -0.23 & 0.60 \\
\hline LMII-thematic units & 0.25 & -0.20 & 0.33 & -0.05 & -0.22 & 0.63 \\
\hline LMII-recognition & 0.16 & -0.16 & 0.24 & -0.27 & -0.20 & -0.26 \\
\hline \multicolumn{7}{|l|}{ Executive function } \\
\hline MCST-C & -0.08 & -0.20 & 0.05 & -0.03 & -0.07 & 0.34 \\
\hline MCST-P & 0.04 & -0.17 & -0.09 & 0.12 & 0.10 & -0.21 \\
\hline Verbal fluency & -0.10 & 0.26 & 0.27 & 0.10 & -0.27 & 0.25 \\
\hline TMTA & -0.34 & $0.40 \dagger$ & 0.02 & -0.03 & 0.28 & 0.37 \\
\hline TMTB & -0.40 & $0.47 \dagger$ & -0.37 & $0.42 \dagger$ & 0.10 & 0.02 \\
\hline $\begin{array}{l}{ }^{*} \text { Correlation is significant at } \\
\text { †Correlation is significant at } \\
\text { See tables } 1 \text { and } 2 \text {. } \\
\text { CTR, corrected target recog } \\
\text { logical memory; MCST-C an } \\
\text { respectively; NCs, normal cc }\end{array}$ & $\begin{array}{l}\text { evel (2-ta } \\
\text { evel (2-ta } \\
\text {; DRM, D } \\
\text { P indicate } \\
\text {, Parkin }\end{array}$ & $\begin{array}{l}\text { iger-Mc } \\
\text { ed cate } \\
\text { e; TMT }\end{array}$ & $\begin{array}{l}\text { radigm; } \\
\text { perseve } \\
\text { ng Test. }\end{array}$ & in the & $\begin{array}{l}\text { oecific } r \\
\text { Card Sc }\end{array}$ & \\
\hline
\end{tabular}

persistently taking on this compensatory role. Further studies involving structural (eg, Voxel-based morphometry, VBM) or functional brain imaging measurements to explore this issue are necessary.

Although early-stage patients with PD display a similar pattern during the gist-based memory task with normal ageing, we found that $10.3 \%$ of early-stage patients with PD had gist memory performances that fell below the 5 th centile of NCs. From a clinical perspective, these patients should not be ignored. A comprehensive evaluation of a patient's cognitive status is crucial for planning cognitive training programmes for patients with PD. Future work focusing on educational and medical care programmes specifically tailored to these patients and their caregivers is also required. Moreover, determining whether impaired gist memory is an early predictor of brain alterations in patients with PD remains an interesting topic. One limitation of the present study is that there was a lack of a comprehensive motor evaluation to categorise the patients into different subgroups. Emerging evidence suggests that different motor symptoms might be related to different levels of cognitive function. ${ }^{36} 37$ We hope that further research will give consideration to the motor heterogeneity of patients with PD. Moreover, 'advanced-stage' PD only included stage III patients in the current study; the small sample size of advanced-stage patients with PD might restrict our interpretation of the results, particularly with respect to the lack of an association between the corrected target recognition rate, gist memory performance and item-specific memory performance. Further work assessing more advanced-stage patients with PD to confirm our findings is also needed. Second, the role of executive function in gist and item-specific memory remains a very promising area of research. We know that executive function is a complex construct, and future research should investigate this function in a more comprehensive way, such as monitoring and initiation ability, to specifically determine the relationship between executive function, gist memory performance and item-specific memory performance. Lastly, factors such as education and medication were also weaknesses in this study; specifically, the impact of these factors on gist memory performance has yet to be clarified and requires future research to explore this potential association.

In summary, to the best of our knowledge, this is the first study to investigate the false recognition effect in patients with PD using the DRM paradigm. The results indicate that gist memory function is preserved in the early-stage PD group; however, impaired gist memory develops in advanced-stage patients with PD. These findings suggest that patients in the early stage of PD could use their preserved gist memory as a potential tool during a memory rehabilitation programme. Thus, our findings may be helpful when developing medical care programmes or designing specific memory rehabilitation strategies for patients with PD.

\section{Author affiliations}

${ }^{1}$ Institute of Behavioral Medicine, College of Medicine, National Cheng Kung University, Tainan, Taiwan

${ }^{2}$ Department of Neurology, Kaohsiung Medical University Hospital, Kaohsiung, Taiwan 
${ }^{3}$ Department of Neurology, Chang Gung Memorial Hospital-Linkou Branch, Chang Gung University College of Medicine, Taoyuan, Taiwan ${ }^{4}$ Department of Neurology, National Taiwan University Hospital, College of Medicine, National Taiwan University, Taipei, Taiwan

${ }^{5}$ Department of Psychology, National Taiwan University, Taipei, Taiwan

${ }^{6}$ Department of Neurobiology and Cognitive Science Center, National Taiwan University, Taipei, Taiwan

${ }^{7}$ Department of Psychiatry, National Taiwan University Hospital, College of Medicine, National Taiwan University, Taipei, Taiwan

${ }^{8}$ Graduate Institute of Brain and Mind Sciences, National Taiwan University, Taipei, Taiwan

Acknowledgements The authors are grateful to the participants involved in this study, and for the grant from the Ministry of Science and Technology (MOST), Taipei, Taiwan (MOST 103-2410-H-006-120-MY2, MOST 103-2633-H-006-001 and MOST 104-2633-H-006 -001). The authors declare no conflict of interest.

Contributors R-LY was responsible for the research project: execution; statistical analysis: design, execution, review and critique; manuscript preparation: writing the first draft. C-HT was responsible for statistical analysis: execution, review and critique; manuscript preparation: review and critique. Y-RW was responsible for research project: execution; manuscript preparation: review and critique. R-MW and M-JC were responsible for research project: execution, manuscript preparation: review and critique. M-SH was responsible for research project: conception, organisation, execution; statistical analysis: design, review and critique, manuscript preparation: review and critique.

Funding This research received no specific grant from any funding agency in the public, commercial or not-for-profit sectors.

Competing interests None declared.

Patient consent Obtained.

Ethics approval The study was approved by the ethical committees of the National Taiwan University Hospital and Chang Gung Memorial Hospital.

Provenance and peer review Not commissioned; externally peer reviewed.

Data sharing statement No additional data are available.

Open Access This is an Open Access article distributed in accordance with the Creative Commons Attribution Non Commercial (CC BY-NC 4.0) license, which permits others to distribute, remix, adapt, build upon this work noncommercially, and license their derivative works on different terms, provided the original work is properly cited and the use is non-commercial. See: http:// creativecommons.org/licenses/by-nc/4.0/

\section{REFERENCES}

1. Alexander GE, DeLong MR, Strick PL. Parallel organization of functionally segregated circuits linking basal ganglia and cortex. Annu Rev Neurosci 1986;9:357-81.

2. Yu RL, Wu RM, Tai $\mathrm{CH}$, et al. Neuropsychological profile in patients with early stage of Parkinson's disease in Taiwan. Parkinsonism Relat Disord 2012;18:1067-72.

3. Yu RL, Wu RM, Chiu MJ, et al. Advanced Theory of Mind in patients at early stage of Parkinson's disease. Parkinsonism Relat Disord 2012;18:21-4.

4. Yu RL, Wu RM. Social brain dysfunctions in patients with Parkinson's disease: a review of theory of mind studies. Transl Neurodegener 2013;2:7.

5. Aarsland D, Zaccai J, Brayne C. A systematic review of prevalence studies of dementia in Parkinson's disease. Mov Disord 2005:20:1255-63.

6. Buter TC, van den Hout A, Matthews FE, et al. Dementia and survival in Parkinson disease: a 12-year population study. Neurology 2008:70:1017-22

7. Aarsland D, Andersen K, Larsen JP, et al. Prevalence and characteristics of dementia in Parkinson disease: an 8-year prospective study. Arch Neurol 2003;60:387-92.

8. Flowers K, Pearce I, Pearce J. Recognition memory in Parkinson's disease. J Neurol Neurosurg Psychiatry 1984;47:1174-81.

9. Whittington CJ, Podd J, Kan MM. Recognition memory impairment in Parkinson's disease: power and meta-analyses. Neuropsychology 2000:14:233-46.

10. Reyna VF, Brainerd CJ. Fuzzy-trace theory and false memory: new frontiers. J Exp Child Psychol 1998;71:194-209.
11. Reyna VF, Brainerd CJ. Fuzzy-trace theory: an interim synthesis. Learn Individual Differences 1995;7:1-75.

12. Roediger HL, McDermott KB. Creating false memories: remembering words not presented in lists. J Exp Psychol Learn Mem Cogn 1995;21:803.

13. Deese J. On the prediction of occurrence of particular verbal intrusions in immediate recall. $J$ Exp Psychol 1959;58:17.

14. Chiu MJ, Lin CW, Chen CC, et al. Impaired gist memory in patients with temporal lobe epilepsy and hippocampal sclerosis. Epilepsia 2010;51:1036-42.

15. Hudon C, Belleville S, Souchay C, et al. Memory for gist and detail information in Alzheimer's disease and mild cognitive impairment. Neuropsychology 2006;20:566.

16. Budson A, Sullivan A, Mayer E, et al. Suppression of false recognition in Alzheimer's disease and in patients with frontal lobe lesions. Brain 2002;125:2750-65.

17. Melo B, Winocur G, Moscovitch M. False recall and false recognition: $n$ examination of the effects of selective and combined lesions to the medial temporal lobe/diencephalon and frontal lobe structures. Cogn Neuropsychol 1999;16:343-59.

18. Verfaellie M, Rapcsak SZ, Keane MM, et al. Elevated false recognition in patients with frontal lobe damage is neither a general nor a unitary phenomenon. Neuropsychology 2004;18:94.

19. Cabeza R, Rao SM, Wagner AD, et al. Can medial temporal lobe regions distinguish true from false? An event-related functional MR study of veridical and illusory recognition memory. Proc Natl Acad Sci USA 2001;98:4805-10.

20. Dennis NA, Kim H, Cabeza R. Age-related differences in brain activity during true and false memory retrieval. $J$ Cogn Neurosci 2008;20:1390-402.

21. Schacter DL, Verfaellie M, Pradere D. The neuropsychology of memory illusions: false recall and recognition in amnesic patients. J Mem Lang 1996;35:319-34.

22. Dobbins IG, Foley $\mathrm{H}$, Schacter $\mathrm{DL}$, et al. Executive control during episodic retrieval: multiple prefrontal processes subserve source memory. Neuron 2002;35:989-96.

23. Moscovitch M, Winocur G. Frontal lobes, memory, and aging. Ann N Y Acad Sci 1995;769:119-50.

24. Parkin AJ, Ward J, Bindschaedler C, et al. Recognition following frontal lobe damage: the role of encoding factors. Cogn Neuropsychol 1999;16:243-65.

25. Verfaellie M, Schacter DL, Cook SP. The effect of retrieval instructions on false recognition: exploring the nature of the gist memory impairment in amnesia. Neuropsychologia 2002;40:2360-8.

26. Davidson PS, Anaki D, Saint-Cyr JA, et al. Exploring the recognition memory deficit in Parkinson's disease: estimates of recollection versus familiarity. Brain 2006;129 7):1768-79.

27. dos Santos CM, Balardin JB, Irigaray TQ, et al. Incidental encoding strategies did not improve contextual memory in Parkinson's disease patients. Neurorehabil Neural Repair 2010;24:450-6.

28. Algarabel S, Rodríguez LA, Escudero J, et al. Recognition by familiarity is preserved in Parkinson's without dementia and Lewy-Body disease. Neuropsychology 2010;24:599-607.

29. Hughes AJ, Daniel SE, Kilford L, et al. Accuracy of clinical diagnosis of idiopathic Parkinson's disease: a clinico-pathological study of 100 cases. J Neurol Neurosurg Psychiatry 1992;55:181-4.

30. Hoehn MM, Yahr MD. Parkinsonism: onset, progression and mortality. Neurology 1967;17:427-42.

31. Folstein MF, Folstein SE, McHugh PR. "Mini-mental state." A practical method for grading the cognitive state of patients for the clinician. J Psychiatr Res 1975;12:189-98.

32. Nelson HE. A modified card sorting test sensitive to frontal lobe defects. Cortex 1976;12:313-24.

33. Hua MS, Chang SH, Chen ST. Factor structure and age effects with an aphasia test battery in normal Taiwanese adults. Neuropsychology 1997;11:156-62.

34. Reitan RM, Wolfson D. The Halstead-Reitan neuropsychological test battery: theory and clinical interpretation. Reitan Neuropsychology, 1985

35. Hua MS, Chang BS, Lin KN, et al. Wechsler memory scale. 3rd edn. [Manual]. Taipei: Chinese Behavioral Science Corp, 2005.

36. Isaacs D, Herb J, Van Wouwe N, et al. Parkinson's disease motor phenotype predicts cognitive performance (P5. 264). Neurology 2014;82(10 Suppl):P5. 264

37. Poletti M, Frosini D, Pagni C, et al. The relationship between motor symptom lateralization and cognitive performance in newly diagnosed drug-naïve patients with Parkinson's disease. J Clin Exp Neuropsychol 2013;35:124-31. 